\title{
SOME NON-ABELIAN EXTENSIONS OF COMPLETELY DIVISIBLE GROUPS ${ }^{1}$
}

\section{FRANKLIN HAIMO}

1. Introduction. Baer $[1 ; 2]$ has showed that those abelian groups $G$ which are direct summands of every including abelian group are precisely those abelian groups $G$ for which $n G=G$ for every positive integer $n$. The latter class of groups consists of the so-called complete or infinitely divisible abelian groups. Examination of the proof of the equivalence of these two classes discloses essential difficulties in the way of extension to the non-abelian case. Once "complete" is suitably defined for these latter groups, we can prove the following: Let $H$ be a complete group interpolated into the ascending central series of a group $G$. Let $K$ be a subgroup, maximal with respect to the property of meeting $H$ on that portion of the ascending series of $G$ below $H$. Then if $N(K)$ is the normalizer of $K$ in $G, N(K)=H+K$. This result seems to be the natural extension of half of the Baer theorem to the non-abelian case.

Let us write all groups additively, whether they be abelian or not. For a subgroup $H$ of a group $G$ we let $N(H ; G)$ be the normalizer of $H$ in $G . C_{v}$ is to be the cyclic subgroup of $G$ generated by the element $v \in G$. $C(v ; G)$ is to be the centralizer of $v$ in $G$. For a subgroup $H$, $C(H ; G)$ is to denote the centralizer of $H$ in $G$. (See [4] for definitions.) Let 0 be the unity of $G$, and let (0) be the one element subgroup of $G$. In what follows, $m, n$, and $r$ will always denote nonzero integers. $D(H ; G)$, for a subset $H$ of $G$, is to be the set of all $x \in G$ for which there exists $m=m(x)$ with $m x \in H . D(H ; G)$, the division-hull of $H$ in $G$, need not be a subgroup of $G$ in the non-abelian case even if $H$ is a subgroup.

We shall say that a group $G$ is complete if, to each ordered pair $(g, n)$, where $g \in G$, there exists a finite set of elements $g_{i}(g, n)=g_{i}$ $(i=1,2, \cdots, m(g, n)=m)$ with $n g_{1}+n g_{2}+\cdots+n g_{m}=g$. If $G$ is both abelian and complete we can always choose $m=1$.

For a group $G$, define in the customary fashion [4] the ascending central series $\left\{Z_{i}(G)\right\}(i=0,1,2, \cdots)$, where $Z_{0}(G)=(0), Z_{1}(G)$ is the center of $G$, and $Z_{i+1}(G) / Z_{i}(G)$ is the center of $G / Z_{i}(G)$. A subgroup $H$ of $G$ is said to be interpolated in the ascending central series at $d$ if $Z_{d}(G) \subset H \subset Z_{d+1}(G)$ (where $\subset$ does not preclude equality), and $d$ is the least integer for which this is true.

Received by the editors January 5, 1953 and, in revised form, May 8, 1953.

1 Research sponsored in part by the OSR, USAF. 
If $H$ and $K$ are subsets of a group $G$, let $H+K$ be the set of all $h+k$, where $h \in H$ and $k \in K$. In general, $H+K$ need not be a subgroup, though it surely is a subgroup if both $H$ and $K$ are and at least one of these is normal. We note here that $\oplus$ denotes direct summation of subgroups.

\section{The normalizer decomposition.}

Lemma 1. Let $H$ and $K$ be a pair of subgroups of $G$ where $K$ is max$i$ mal with respect to the property of being disjoint from $H$. Then $N(K ; G)$ $C D(H+K ; G)$.

Proof. We follow [1] and [3]. If $x \in N(K ; G), x \in H+K$, then $x \notin K$. Form the subgroup $K^{\prime}=\{K, x\}$ which has the generators $x$ and all the elements of $K$. $K^{\prime}$ includes $K$ properly. By the maximal character of $K$, one can find a nonzero element $h \in K^{\prime} \cap H$. Since $x \in N(K ; G)$, it is possible to find an integer $t$ and an element $k \in K$ with $h=t x+k$. If $t=0$, then $h=k$; and $H \cap K=(0)$ then implies $h=0$, a contradiction. Hence $t x \in H+K$ with nonzero $t$, and $x \in D(H+K ; G)$. Since also $(H+K) \cap N(K ; G) \subset D(H+K ; G)$, it follows that $N(K ; G)$ $\subset D(H+K ; G)$.

THEOREM 1. Let $H$ be a complete group interpolated at $d$ into the ascending central series of a group $G$. Let $K$ be a subgroup of $G$, maximal with respect to the property of having precisely $Z_{d}(G)$ as its intersection with $H$. Then $N(K ; G)=H+K$ and $N(K ; G) / Z_{d}(G) \cong H / Z_{d}(G)$ $\oplus K / Z_{d}(G)$.

Proof. We follow [3]. Suppose that $d=0$. Then $H$ is included in the center of $G$, and $H \cap K=(0)$. For a given $x \in N(K ; G), x \in H+K$, let $r$ be the least positive integer (provided by Lemma 1) for which $r x \in H+K$. Let $p$ be a prime divisor of $r$, and let $y=(r / p) x$. $p y=r x$ $=h+k$ for suitable $h \in H$ and $k \in K$. Since $H$ is complete and abelian, there exists $h_{1} \in H$ with $p h_{1}=h$; and $-p h_{1}+p y=k$. Since also $h_{1} \in Z_{1}(G), \quad-p h_{1}+p y=p\left(-h_{1}+y\right)=k$. Let $z=-h_{1}+y$. Since $H$ $\subset Z_{1}(G)$ and since $y=(r / p) x \in N(K ; G)$, it follows that $z \in N(K ; G)$. If $z \in H+K$, then $y=(r / p) x \in H+K$, contradicting the minimum character of $r$. Form the subgroup $K^{\prime \prime}=\{K, z\}$. $K^{\prime \prime}$ includes $K$ properly, and by the maximal character of the latter subgroup there exists a nonzero $h^{\prime} \in K^{\prime \prime} \cap H$. Since $z \in N(K ; G)$ we can find an integer $t$ and an element $k^{\prime} \in K$ with $h^{\prime}=t z+k^{\prime}$. If $p \mid t, p z \in K$ implies $h^{\prime} \in K$, contradicting $H \cap K=(0)$. Then there exist integers $a$ and $b$ for which $a t+b p=1$, so that $z=a t z+b p z$. But $t z \in H+K$ implies atz $\in H+K$ since $H \subset Z_{1}(G)$; and $p z \in K$. Thus $z \in H+K$, a contradiction. We 
have established that $r=1, x \in H+K$, and that $N(K ; G)=H+K$ if $d=0$.

If $d \neq 0$, reduce the group modulo $Z_{d}(G)$. Let the images of $G, H$, and $K$ be, respectively, $G^{\prime}, H^{\prime}$, and $K^{\prime}$. It can be readily checked that $K^{\prime}$ is maximal in $G^{\prime}$ with respect to the property of being disjoint from $H^{\prime}$, that $H^{\prime}$ is in the center of $G^{\prime}$, and that $H^{\prime}$ is a complete abelian group. Using the case $d=0$ above, we have $N\left(K^{\prime} ; G^{\prime}\right)=H^{\prime}$ $+K^{\prime}$, and a trivial argument now shows that $N(K ; G)=H+K$.

Subgroups which are interpolated into the ascending central series are normal subgroups, so that $H$ is normal in $G$, and $H / Z_{d}(G)$ is normal in $G / Z_{d}(G)$. Hence $H / Z_{d}(G)$ is normal in $N(K ; G) / Z_{d}(G)$. Moreover $K$ is normal in $N(K ; G)$ so $K / Z_{d}(G)$ is normal in $N(K ; G) / Z_{d}(G)$. Since $H / Z_{d}(G) \cap K / Z_{d}(G)=(0)$, and since $N(K ; G) / Z_{d}(G)=H / Z_{d}(G)$ $+K / Z_{d}(G)$, we have proved that the sign + in the last statement can be replaced by $\oplus$.

An immediate result is

Corollary 1. Let a complete group $H$ have an extension to a nilpotent group $G$ of class $d+1$ in such a way that $Z_{d}(G) \subset H$. If $K$ is any subgroup of $G$ which is maximal with respect to the property of having precisely $Z_{d}(G)$ as its intersection with $H$, then $K$ is normal in $G$, and $G=H+K$.

Corlllary 2. If $H$ and $K$ are as in the theorem and if $d=0$, then $N(K ; G) \cong H \oplus K$.

Corollary 3. If $H$ and $K$ are as in the theorem and if $d=0$, then $N(K ; G) / C(K ; G) \cong J(K)$, the group of inner automorphisms of $K$.

Proof. Since $C(K ; G) \subset N(K ; G)$ and since $N(K ; G)=H+K$, every element of $C(K ; G)$ has the form $h+k$, where $h \in H$ and $k \in K$. $h+k+k^{\prime}=k^{\prime}+h+k$ for every $k^{\prime} \in K$. Since $H \subset Z_{1}(G), k+k^{\prime}=k^{\prime}+k$ for every $k^{\prime} \in K$, and $k \in Z_{1}(K)$. We can thus establish that $C(K ; G)$ $=H+Z_{1}(K)$. For $k \in K$, let $\gamma_{k}$ be the inner automorphism $\gamma_{k}(x)$ $=k+x-k$ for every $x \in K$. Define a map $\theta$ on $H+K$ into $J(K)$ as follows: $\theta(h+k)=\gamma_{k}$. Then it is easy to verify that $\theta$ is a homomorphism on $H+K$ onto $J(K)$ with kernel $H+Z_{1}(K)$.

One could ask whether there is anything to be said if $H$ is a subgroup not necessarily complete or interpolated into the ascending central series. Let (A) be the property of a proper subgroup $H$ of a group $G$ that $m u+v$ (or, alternately, $v+m u) \in H$ where $u, v \in G$ and $C_{v} \cap H=(0)$ implies the existence of $g \in H \cap C(u ; G)$ with $m g=m u+v$ (alternately, $v+m u$ ). Suppose that $G$ is aperiodic and that $H$ is a proper subgroup of $G$ with property (A). Then it is easy to prove that 
$H$ is a normal subgroup of $G$ and that $D(H ; G)=H \subset \cap C(u ; G)$, where the cross-cut is taken over all elements $u \in G$ such that $u \notin H$. $H$ is likewise strongly complete in the sense that the equation $n x$ $=h \in H$ always has a solution in $H$. Even if $G$ has nontrivial periodic elements, a strongly complete subgroup $H$ which is included in the center of $G$ has property (A). The proofs of Lemma 1 and of Theorem 1 can be rewritten to give the somewhat weaker result:

Theorem 2. Let $H$ be a subgroup with property (A) in a group $G$. Suppose that there exists a normal subgroup $K$ of $G$ which, as a subgroup, is maximal among the set of all subgroups (normal or not) which are disjoint from $H$. Then $G=H+K$; and if $G$ is aperiodic, $G=H \oplus K$.

\section{BIBLIOGRAPHY}

1. R. Baer, The subgroup of the elements of finite order of an abelian group, Ann. of Math. (2) vol. 37 (1936) pp. 766-781.

2. - Abelian groups that are direct summands of every containing abelian group, Bull. Amer. Math. Soc. vol. 46 (1940) pp. 800-806.

3. I. Kaplansky, Infinite Abelian groups, The University of Chicago, mimeographed, 81 pp., n.d.

4. H. Zassenhaus, Lehrbuch der Gruppentheorie I, Leipzig and Berlin, 1937.

WASHINGTON UNIVERSITY 